\title{
Short-Term Effects of Sublethal Doses of Nematicides on Meloidogyne incognita
}

\author{
Catherine L. Wram ${ }^{1, \dagger}$ and Inga A. Zasada ${ }^{2}$ \\ ${ }^{1}$ Department of Botany and Plant Pathology, Oregon State University, Corvallis, OR 97331 \\ ${ }^{2}$ Horticultural Crops Research Unit, U.S. Department of Agriculture Agricultural Research Service, Corvallis, OR 97331 \\ Accepted for publication 24 April 2019.
}

\begin{abstract}
Meloidogyne species are one of the most important groups of plantparasitic nematodes globally because of their ability to damage most cultivated plants. Although they are widespread and economically important, there are limited control measures to combat these nematodes. New nonfumigant nematicides have been discovered that have the potential to be widely utilized for the management of plant-parasitic nematodes. Because of the longer half-lives in soil and lower toxicity of new nematicides compared with traditional fumigant and nonfumigant nematicides, understanding how nematodes respond to sublethal doses of nematicides is imperative to understanding whether nematicide resistance has the potential to develop. Characterizing responses of nematodes to sublethal doses will provide the foundation for future work, such as gene expression studies. In this study, the nematicides oxamyl (Vydate), fluazaindolizine (Salibro), fluensulfone (Nimitz), and fluopyram (Velum), were evaluated to understand how sublethal doses affect the fecundity and mobility of Meloidogyne incognita second-stage juveniles (J2). Using a microwell assay system, doseresponse curves for each nematicide were established for $M$. incognita $\mathrm{J} 2$.

predicted $\mathrm{ED}_{50}$ values (effective doses that resulted in $50 \%$ of the population becoming inactive) of 89.4, 131.7, and $180.6 \mathrm{ppm}$ for oxamyl, fluensulfone, and fluazaindolizine, respectively. The 24-h $\mathrm{ED}_{50}$ of each nematicide was then used in both motility and infectivity assays. The motility and activity of M. incognita $\mathrm{J} 2$ exposed to $\mathrm{ED}_{50}$ doses of fluazaindolizine and fluensulfone was significantly reduced, with nematodes initially being motile but eventually becoming inactive. However, the motility of M. incognita J2 exposed to $\mathrm{ED}_{50}$ doses of fluopyram and oxamyl was not different from a water control. In a pot assay, $M$. incognita $\mathrm{J} 2$ exposed to $\mathrm{ED}_{50}$ doses of fluazaindolizine, oxamyl, and fluensulfone were unable to reproduce on tomato, with reproduction factors $(\mathrm{RF}=$ final population density/initial population density) of 0 to 0.03 . Fluopyram did not reduce reproduction of M. incognita, with a mean RF of $38.7 \pm 4.5$, which was similar to the RF of $46.3 \pm 4.6$ for the water control. This study is the first comprehensive evaluation of $M$. incognita activity, motility, and fecundity after exposure to the traditional nematicide, oxamyl, as well as three new nematicides, fluazaindolizine, fluopyram, and fluensulfone.
\end{abstract} Fluopyram was the most toxic nematicide, with effective doses up to 230 times lower than that of other nematicides. The other nematicides had

Plant-parasitic nematodes (PPNs) cause damage to a wide variety of agricultural crops, resulting in yield and quality losses of up to $\$ 118$ billion yearly (Bernard et al. 2017). Of the PPNs, root-knot nematodes of the Meloidogyne species are the most important genera (Jones et al. 2013). The permanent feeding sites established in roots by these sedentary endoparasitic species during the parasitism process reduces the plant's ability to uptake nutrients and water from the soil. This leads to stunting of plant growth, wilting during mild water stress, and chlorosis (Bernard, et al. 2017; Perry and Moens 2013). The significant economic impact of Meloidogyne spp. is attributable to their ability to infect almost all cultivated plants and to their worldwide distribution. Of the species in this genus, M. incognita is the most prevalent and destructive (Mitkowski and Abawi 2003; Perry and Moens 2013; Trudgill and Blok 2001). M. incognita is found in 29 states in the United States, is widespread in 20 countries in Africa, and is endemic in subtropical and tropical regions around the globe (CABI 2018).

†Corresponding author: C. Wram; wramc@oregonstate.edu

Funding: Financial support was provided by Corteva Agriscience and by the U.S. Department of Agriculture Agricultural Research Service Current Research Information System (2072-22000-043-00D).

Mention of trade names or commercial products in this publication is solely for the purpose of providing specific information and does not imply recommendation or endorsement by the U.S. Department of Agriculture.

The author(s) declare no conflict of interest.

C 2019 The American Phytopathological Society
Keywords: disease control, pest management

To manage PPNs, common control methods like plant resistance and trap crops can be used, but the most reliable methods of control are often chemical, including fumigates and nonfumigant nematicides (Zasada et al. 2010). Almost half of the $\$ 1$ billion global nematicide market is used to control Meloidogyne species (Perry and Moens 2013). Traditionally, fumigants such as methyl bromide, chloropicrin, and 1,3-dichloropropene have been heavily relied on to manage PPNs, soilborne pathogens, and weeds, owing to their broad-spectrum activity and efficacy. Other conventionally used nonfumigant nematicides include organophosphates like fosthiazate and fenamiphos and carbamates like oxamyl (Zasada et al. 2010). Both organophosphates and carbamates act as acetylcholinesterase inhibitors; their toxicity is not limited to nematodes and can be hazardous to humans and insects (Costa et al. 2008). Both methyl bromide and oxamyl have relatively short soil half-lives of 5 and 30 days, respectively (Osman et al. 2009; Yates et al. 2002). Because of harmful environmental effects, potential health hazards to workers, and/or limited supply, many of these conventional chemical products have been eliminated or are restricted (Duniway 2002; Martin 2003; U.S. Environmental Protection Agency 2008; Zasada et al. 2010). There have been few new nematicides to fill this gap in PPN management until recently in the past 5 years, with the development of fluopyram (Velum Prime), fluensulfone (Nimitz), and fluazaindolizine (Salibro).

Of these new nonfumigant nematicides, only one has a known mode of action, fluopyram. Fluopyram, also a Fungicide Resistance Action Committee code 11 fungicide, is an inhibitor of the enzyme succinate dehydrogenase and was registered for use in cotton and peanut for the control of PPNs in 2015 (Faske and Hurd 2015). Fluopyram persists much longer in the environment than traditional 
nematicides, with a half-life of up to 746 days depending on soil type (Ludlow 2015a).

Fluensulfone, a heterocyclic fluoroalkenyl sulfone, has an unknown but novel mode of action (Kearn et al. 2014). Fluensulfone is registered for use on a variety of crops from leafy and fruiting vegetables to root vegetables and tree nuts to suppress PPNs such as Meloidogyne spp., Globodera spp., and Pratylenchus spp. Fluensulfone suppressed G. pallida and Meloidogyne spp. in a variety of crop species, including potatoes, tomatoes, lima beans, and other vegetable crops (Castillo et al. 2017; Jones et al. 2017; Kearn et al. 2017; Morris et al. 2015, 2016). Caenorhabditis elegans and $M$. incognita responded similarly when exposed to similar doses of fluensulfone, with the compound resulting in immobility and death. However, C. elegans mutants resistant to organophosphates and carbamates were susceptible to fluensulfone, indicating that cholinesterase inhibition is not the target for this compound (Kearn et al. 2014). Fluensulfone has a half-life in soil up to 36 days (Ludlow 2015b).

Fluazaindolizine, an imidazopyridine that is still in the process of registration, is a new nematicide with a novel mode of action different from that of carbamates and organophosphates (Lahm et al. 2017). Inhibition of motility and mortality of C. elegans adults and Drosophila melanogaster egg and adult stages was not observed at concentrations of 200 and $300 \mathrm{ppm}$, respectively. However, $M$. incognita exposed to 5 to $50 \mathrm{ppm}$ of fluazaindolizine were increasingly immobile and dead at 24 to $96 \mathrm{~h}$ after exposure (Lahm et al. 2017). This indicates that the target of fluazaindolizine is PPN specific.

Nonfumigant nematicides are applied in granular or liquid form and move through the soil via percolation. Effective PPN control with nematicides is only accomplished if a lethal dose of the compound is maintained for the right exposure period. There are many factors that affect nematicide movement and retention in soil, including soil type, affinity of the nematicide to organic matter, mobility of the nematicide, the amount of water applied with the nematicide, speed of microbial degradation, and half-life of the nematicide in the soil (Noling 1997). These variables create a soil environment where sublethal doses are likely to occur and therefore, PPNs will be exposed to a range of concentrations of a compound. This variable dose exposure of nematicides to PPNs has been demonstrated for oxamyl (Haydock et al. 2012). Oxamyl degraded at different rates depending on soil temperature, soil moisture, and soil type. In this study, an increase in soil temperature and loamy sand soil type led to an increase in oxamyl degradation, whereas low soil moisture led to oxamyl retention. The soil dynamics listed above and the longer half-lives of newer nematicides (ranging from 32 to $>700$ days) combine to create an environment where PPNs have the potential to be exposed to nematicides at sublethal concentrations. Therefore, the goal of this research was to characterize the short-term effects of sublethal concentrations of nematicides on $M$. incognita in order to create a foundation for further work aimed at understanding the underpinnings of how these nematicides affect nematode biology. The objectives of this study were as follows: (i) to develop dose-response curves for the nematicides using $M$. incognita, (ii) to evaluate the effect that sublethal doses of nematicides have on $M$. incognita motility, and (iii) to assess the effect of sublethal doses of nematicides on $M$. incognita fecundity. The nematicides oxamyl (Vydate), fluensulfone (Nimitz), fluopyram (Velum Prime), and fluazaindolizine (Salibro) were evaluated.

\section{MATERIALS AND METHODS}

Nematode inoculum. An $M$. incognita population originally collected from grape (Vitis vinifera) in Parlier, California, was used in all experiments. To establish the population in culture, a single egg mass was placed on the roots of tomato (Solanum lycopersicum 'Rutgers'). After 6 to 8 weeks, additional tomato plants were inoculated with egg masses hand-picked from the plant inoculated with a single egg mass. M. incognita second-stage juveniles (J2) were collected by hand picking egg masses from established culture tomato roots and placing the masses on a $1.5-\mathrm{cm}$-diameter plastic hatching chamber with a 30- $\mu \mathrm{m}$ nylon mesh in a 40-ml beaker containing water (Nitao et al. 1999; Zasada et al. 2006). Hatched $M$. incognita $\mathrm{J} 2$ were collected after 3 days and stored at $4^{\circ} \mathrm{C}$ until used (no more than 2 days). $M$. incognita eggs were obtained by covering infected tomato roots with $0.6 \%$ sodium hypochlorite and agitating for $3 \mathrm{~min}$ at $300 \mathrm{rpm}$. Working in a laminar flow hood, the roots were rinsed with autoclaved deionized water over sterilized, nested 250- and 25.4- $\mu \mathrm{m}$ sieves, with eggs collected from the latter.

Microwell dose-response assays. Formulated product was used in all assays. Therefore, all concentrations shown are those of the formulated product, not the active ingredient (a.i.). The percentage of active ingredient is as follows for each product tested: Vydate-L, 24.0\% oxamyl; Nimitz, $40.0 \%$ fluensulfone; Velum Prime, $41.5 \%$ fluopyram; and Salibro, $50.0 \%$ fluazaindolizine. To establish dose-response curves of the short-term effects on $M$. incognita $\mathrm{J} 2$ for each nematicide, microwell assays were conducted (Meyer et al. 2008; Zasada et al. 2006). In each well of a 24-well cell culture plate (Falcon; Corning, Corning, NY), 50 to 60 M. incognita $\mathrm{J} 2$ suspended in $100 \mu \mathrm{l}$ of water were pipetted into $900 \mu \mathrm{l}$ of nematicide solution. Each microwell plate contained a single nematicide at five concentrations, with a water control, replicated four times. Preliminary studies identified an effective range of concentrations for each compound, which were then used to identify nematicide concentrations to test to generate doseresponse curves (data not shown). The plates were sealed with parafilm (Parafilm; Bemis Company, Neenah, WI), covered with foil, and incubated at room temperature for $24 \mathrm{~h}$, a time point used in previous studies to represent short-term effects (Faske and Hurd 2015; Oka 2014). After the incubation period, the plate was placed under 100× magnification on an inverted microscope (Leica DM IL; Leica Microsystems, Wetzlar, Germany) and the number of active versus inactive $M$. incognita was determined. Inactive nematodes were straight and not moving, whereas moving nematodes were considered active even if they exhibited abnormal movements (sluggishness, twitching, etc.). The nematicide solution was then removed from each well using a pipette and replaced with $1 \mathrm{ml}$ of water to establish whether the effects of the nematicides were reversible. After another 24-h incubation at room temperature, the number of active versus inactive nematodes was determined as described above. Each microwell assay was repeated at least two times encompassing a range of concentrations for each nematicide. The concentrations tested for each nematicide were as follows: fluazaindolizine, 210 to $460 \mathrm{ppm}$; fluopyram, 0.5 to $4 \mathrm{ppm}$; fluensulfone, 25 to $700 \mathrm{ppm}$; and oxamyl, 60 to $140 \mathrm{ppm}$.

Experiments to evaluate the effects of nematicides on egg hatch of $M$. incognita were also conducted using the same microwell assay system described above. To each well, 100 eggs suspended in $100 \mu \mathrm{l}$ of water were added to nematicide solutions. The concentrations tested varied for each nematicide but represented a range of effective concentrations for each nematicide as established by the $M$. incognita $\mathrm{J} 2$ dose-response assays. The 24 -well plates were sealed with parafilm, covered, and incubated at room temperature for 7 days. The number of hatched $\mathrm{J} 2$ and the percentage active $\mathrm{J} 2$ were then determined using the same methodology described above. Each assay was conducted at least twice and concentrations were replicated four times.

Motility assays. To determine the effects of sublethal doses of fluazaindolizine, oxamyl, fluensulfone, and fluopyram on the movement of $M$. incognita $\mathrm{J} 2$, motility assays were conducted. In a 1.7-ml microcentrifuge tube, $500 \mathrm{M}$. incognita $\mathrm{J} 2$ were suspended in $100 \mu \mathrm{l}$ of water and treated with $900 \mu \mathrm{l}$ of a nematicide solution. The nematicide solution was equivalent to an $\mathrm{ED}_{50}$ dose (effective dose needed to cause $50 \%$ of the population to become inactive) of each nematicide established in the $M$. incognita $\mathrm{J} 2$ dose-response 
assays (fluazaindolizine, $230 \mathrm{ppm}$; oxamyl, $100 \mathrm{ppm}$; fluopyram, 1 ppm; and fluensulfone, $95 \mathrm{ppm}$ ). After 2 and $24 \mathrm{~h}$ of exposure, $500 \mu \mathrm{l}$ from each tube was placed on a $30-\mu \mathrm{m}$ mesh hatching sieve, rinsed with water for 5 to $10 \mathrm{~s}$, and placed in a 60- $\times 15-\mathrm{mm}$ Petri dish containing enough water to just cover the mesh (Nitao et al. 1999). The dishes were covered and incubated for $24 \mathrm{~h}$ at room temperature, after which the sieve was removed, the Petri dish was placed under a dissecting microscope (Leica M205 C) at $2 \times$ magnification, and active versus inactive $M$. incognita $\mathrm{J} 2$ were enumerated. Each time point was replicated six times per nematicide and the assay was conducted three times.

Infectivity assays. To test the effects of sublethal doses of nematicides on reproduction of $M$. incognita, potted tomato Rutgers plants were treated with $\mathrm{ED}_{50}$ doses of nematicides. Two- to threeweek-old tomato seedlings were transplanted into a sand/loam mix (1:1) in 10-cm pots (Anderson Pots no. 1007; Anderson Die \& Manufacturing Co., Portland, OR). Pots were then drenched with $145 \mathrm{ml}$ of nematicide solution (fluazaindolizine, fluensulfone, fluopyram, or oxamyl at an $\mathrm{ED}_{50}$ dose indicated above for motility assay) or water. Immediately after drenching, plants were inoculated with 2,500 M. incognita $\mathrm{J} 2$ suspended in $3 \mathrm{ml}$ of water, by pipetting the solution containing nematodes into two holes $4.5 \mathrm{~cm}$ deep on either side of the plant into the root zone. Each nematicide treatment and the water control were replicated six times and the experiment was conducted twice. Inoculated plants were arranged in a randomized design in a greenhouse and grown under long-day conditions (16-h photoperiod) with $26 / 18^{\circ} \mathrm{C}$ day/ night temperatures. After 1 week, plants were fertilized using a 9N-45P-15K plant starter fertilizer at the labeled rate (Jack's Professional Plant Starter; JR Peters Inc., Allentown, PA). Thereafter, plants were watered twice a week with $20 \mathrm{~N}-20 \mathrm{P}-20 \mathrm{~K}$ fertilizer at the labeled rate (JR Peters Inc.). After 6 to 7 weeks, plants were destructively harvested. The tops were removed and discarded, and roots were gently washed with water to remove adhering soil. Eggs were extracted by placing rinsed roots in $0.6 \%$ sodium hypochlorite and agitating at $300 \mathrm{rpm}$ for $3 \mathrm{~min}$. Roots were then rinsed over nested 250- and 25.4- $\mu \mathrm{m}$ sieves, with eggs collected from the latter. Eggs were enumerated using an inverted microscope as described above. Roots were dried in a $65^{\circ} \mathrm{C}$ oven for 7 days, after which dry roots were weighed.

Statistical analyses. All statistical analyses were conducted using R software (R Core Team 2017). A dose-response curve model was created for $M$. incognita $\mathrm{J} 2$ using the R package $d r c$ (Ritz et al. 2015); specifically, a three-parameter log-logistic model was used for the dose-response curves of oxamyl and fluensulfone, and a four parameter log-logistic model was used for fluopyram and fluazaindolizine. The microwell assay data were transformed using a Box-Cox transformation to correct for variance heterogeneity and nonnormality. Each model was then utilized to determine the $\mathrm{ED}_{50}$ concentration in the $d r c$ package following the methodology outlined in Ritz et al. (2015). Data from the egg hatch doseresponse, motility, and infectivity assays were analyzed using the Kruskal-Wallis test and mean separation using the Wilcox rank sum comparisons test with a Bonferroni multiple comparison correction. The reproduction factor (RF) was calculated by dividing the final egg density $(P f)$ by the initial density of $M$. incognita J2 (Pi) (Windham and Williams 1987). All values are presented as the mean \pm standard error.

\section{RESULTS}

Microwell dose-response assays. The proportion of active $M$. incognita $\mathrm{J} 2$ after 24-h exposure and after the compound was removed and replaced with water was modeled using a log-logistic regression model with four potential parameters (Table 1). The parameters were as follows: $b$, the coefficient denoting steepness of the dose-response curve; $c$ and $d$, the lower and upper asymptotes of the response curve, respectively; and $e$, the effective dose needed to cause $50 \%$ of the population to become inactive $\left(E_{50}\right)$ (Ritz et al. 2015). The models for fluazaindolizine and fluopyram were modeled using all four parameters. However, the models for fluensulfone and oxamyl were made using a three-parameter model, with $c$ (the lower asymptote parameter) fixed at 0 .

Fluopyram had the smallest range of concentrations in the doseresponse curves (Table 2), 100 times smaller than that of the other compounds, making fluopyram the most toxic of the nematicides evaluated. However, this nematicide, behaved like a nemastatic compound in the short term (Fig. 1A). When the compound was removed and replaced with water, the proportion of $M$. incognita $\mathrm{J} 2$ that recovered was between 12.5 and $275 \%$ with increasing concentrations.

The dose-response curve for fluensulfone had the shallowest slope of the four curves (Fig. 1B). Fluensulfone behaved like a true nematicide in the short term, with $<20 \%$ of $M$. incognita $\mathrm{J} 2$ recovering after exposure to the nematicide. Fluensulfone had a predicted $\mathrm{ED}_{50}$ intermediate to oxamyl and fluazaindolizine (Table 2).

Of the four nematicides, oxamyl had the steepest slope parameter, approximately four times larger than the other three compounds

TABLE 1. Dose-response curve log-logistic equation parameters for Meloidogyne incognita second-stage juveniles exposed to nematicides

\begin{tabular}{|c|c|c|c|c|c|}
\hline \multirow[b]{2}{*}{ Nematicide } & \multirow[b]{2}{*}{ Exposure period } & \multicolumn{4}{|c|}{ Model parameter } \\
\hline & & $b^{\mathrm{w}}$ & $c^{\mathrm{x}}$ & $d$ & $e^{\mathrm{y}}$ \\
\hline \multirow[t]{2}{*}{ Fluopyram } & $24 \mathrm{~h}$ & $1.68(0.42)^{\mathrm{z}}$ & $7.64(12.98)$ & $98.53(2.99)$ & $1.31(0.29)$ \\
\hline & & $P=0.233$ & $P=0.00045$ & $P<0.0001$ & $P=0.029$ \\
\hline \multirow[t]{2}{*}{ Oxamyl } & $24 \mathrm{~h}$ & $8.24(1.45)$ & Fixed & $80.42(6.69)$ & $89.34(4.56)$ \\
\hline & & $P<0.0001$ & & $P<0.0001$ & $P<0.0001$ \\
\hline \multirow[t]{4}{*}{ Fluensulfone } & $24 \mathrm{~h}$ & $0.90(0.07)$ & Fixed & $94.14(3.03)$ & $131.72(12.81)$ \\
\hline & & $P<0.0001$ & & $P<0.0001$ & $P<0.0001$ \\
\hline & $24 \mathrm{~h}$ after replacement & $2.26(0.21)$ & Fixed & $94.92(3.06)$ & $13.54(1.75)$ \\
\hline & & $P<0.0001$ & & $P<0.0001$ & $P<0.0001$ \\
\hline \multirow[t]{3}{*}{ Fluazaindolizine } & $24 \mathrm{~h}$ & $2.57(1.07)$ & $41.78(5.88)$ & $91.80(2.82)$ & $180.59(20.18)$ \\
\hline & & $P=0.0176$ & $P<0.0001$ & $P<0.0001$ & $P<0.0001$ \\
\hline & $24 \mathrm{~h}$ after replacement & $1.76(0.45)$ & $-20.61(16.86)$ & $89.19(2.76)$ & $245.33(45.89)$ \\
\hline
\end{tabular}

\footnotetext{
${ }^{\mathrm{w}} b$ is the coefficient denoting steepness of the dose-response curve.

${ }^{\mathrm{x}} c$ and $d$ are the lower and upper asymptotes of the response curve, respectively, fixed to zero in some models.

y $e$ is the effective dose needed to cause $50 \%$ of the population to become inactive.

$\mathrm{z}$ Model parameter, followed by the standard error in parentheses and the $P$ value for the parameter.
} 
(Table 1). Once past $60 \mathrm{ppm}$, even a small increase in dosage resulted in a sharp decrease in the proportion of active $M$. incognita $\mathrm{J} 2$ after 24-h exposure. Oxamyl was nematicidal in the short-term assay (Fig. 1C), with no recovery of $M$. incognita $\mathrm{J} 2$ even after the compound was removed and replaced with water.

Fluazaindolizine also acted as a true nematicide (Fig. 1D). $M$. incognita $\mathrm{J} 2$ continued to die after the compound was replaced

TABLE 2. Comparison of nematicide doses that resulted in $50 \%$ of the population of Meloidogyne incognita second-stage juveniles becoming inactive $\left(\mathrm{ED}_{50}\right)$ in microwell assays

\begin{tabular}{lccc}
\hline Nematicide & \% a.i.y & $\begin{array}{c}\text { Model predicted } \\
\mathrm{ED}_{50}(\mathrm{ppm})^{\mathrm{z}}\end{array}$ & $\begin{array}{c}\mathrm{ED}_{50} \text { used in mobility } \\
\text { and infectivity assays } \\
(\mathrm{ppm})\end{array}$ \\
\hline Fluopyram & 41.5 & $1.30(0.74-1.88)$ & 1 \\
Oxamyl & 24.0 & $89.39(80.33-98.45)$ & 100 \\
Fluensulfone & 40.0 & $131.72(106.52-156.92)$ & 95 \\
Fluazaindolizine & 50.0 & $180.59(140.81-220.37)$ & 230 \\
\hline
\end{tabular}

y Percentage of active ingredient of formulated nematicide.

$\mathrm{z}$ The $95 \%$ confidence interval for each $\mathrm{ED}_{50}$ is enclosed in parentheses. with water, with the proportion of active $M$. incognita $\mathrm{J} 2$ decreasing 18.8 to $100 \%$ with increasing concentrations. The model predicted the highest $24-\mathrm{HD}_{50}$ for fluazaindolizine of the nematicides tested (Table 2).

The effects of fluopyram, oxamyl, fluensulfone, and fluazaindolizine on $M$. incognita egg hatch and activity of hatched $\mathrm{J} 2$ are shown in Figure 2. Only the highest concentration of fluopyram tested $(2.5 \mathrm{ppm})$ reduced $M$. incognita egg hatch compared with the water control (Fig. 2A). Of the M. incognita J2 that hatched, fluopyram concentrations $\geq 1.0 \mathrm{ppm}$ had significantly fewer active $M$. incognita $\mathrm{J} 2$ compared with the water control (Fig. 2E). At the concentration of fluopyram identified as the $24-\mathrm{h} \mathrm{ED}_{50}(1 \mathrm{ppm})$, there was no difference from the control in the mean percentage of $M$. incognita eggs that hatched; however, the mean percentage of active hatched $M$. incognita $\mathrm{J} 2$ was $44 \%$ lower than the water control $(P=0.017)$.

Oxamyl reduced $M$. incognita egg hatch at concentrations $\geq 50 \mathrm{ppm}$ compared with the water control (Fig. 2B). The same trend was observed in the mean percentage of active hatched $M$. incognita $\mathrm{J} 2$ (Fig. 2F). The 24-h $\mathrm{ED}_{50}$ for oxamyl $(100 \mathrm{ppm})$ reduced egg hatch
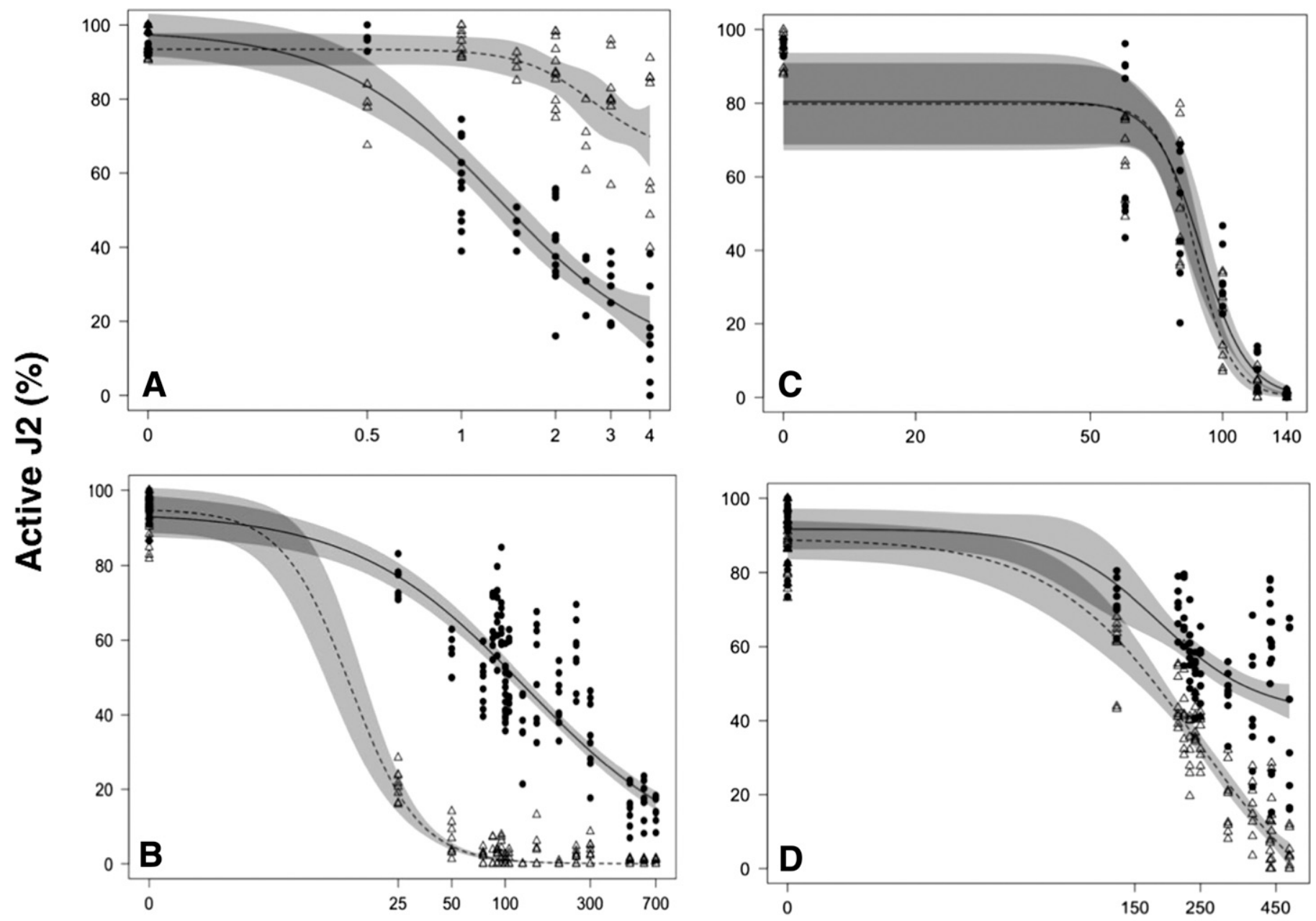

\section{Concentration (ppm)}

Fig. 1. Dose-response curves of Meloidogyne incognita second-stage juveniles (J2) exposed to nematicides. M. incognita J2 were exposed to A, fluopyram, B, fluensulfone, C, oxamyl, and $\mathbf{D}$, fluazaindolizine at a range of concentrations for $24 \mathrm{~h}$. After $24 \mathrm{~h}$, the percentage of active J2 was determined and the nematicide solution was replaced with water. After another $24 \mathrm{~h}$, the percentage of active $\mathrm{J} 2$ was determined to ascertain whether the effects of the compounds on $M$. incognita J2 were reversible. Dose-response curves were determined for Box-Cox transformed data using a log-logistic model with three parameters for fluensulfone and oxamyl and four parameters for fluazaindolizine and fluopyram. The solid line is the curve representing the first 24-h exposure period, with the solid circles indicating each data point. The dotted line is the model for the period after the nematicide was removed, with the individual data points represented as triangles. In both models, the $95 \%$ confidence interval is represented by the shaded region around the curve. 
compared with the water control by $86 \%(P=0.014)$ and reduced the mean percentage of active hatched $M$. incognita by $93 \%(P=0.005)$.

At all tested concentrations, fluensulfone reduced $M$. incognita egg hatch and percentage of active hatched $M$. incognita J2 compared with the water control $(P<0.0002$; Fig. $2 \mathrm{C}$ and $\mathrm{G})$. Across concentrations, fluensulfone reduced egg hatch by at least
$86 \%$ compared with the water control. A $100 \%$ reduction in the mean percentage of active hatched $M$. incognita $\mathrm{J} 2$ was observed at all concentrations compared with the water control $(P<0.0001)$. The fluensulfone 24-h $\mathrm{ED}_{50}(95 \mathrm{ppm})$ followed these same trends.

Fluazaindolizine was a poor ovicide with effects on M. incognita egg hatch only at the two highest concentrations tested, 390 and
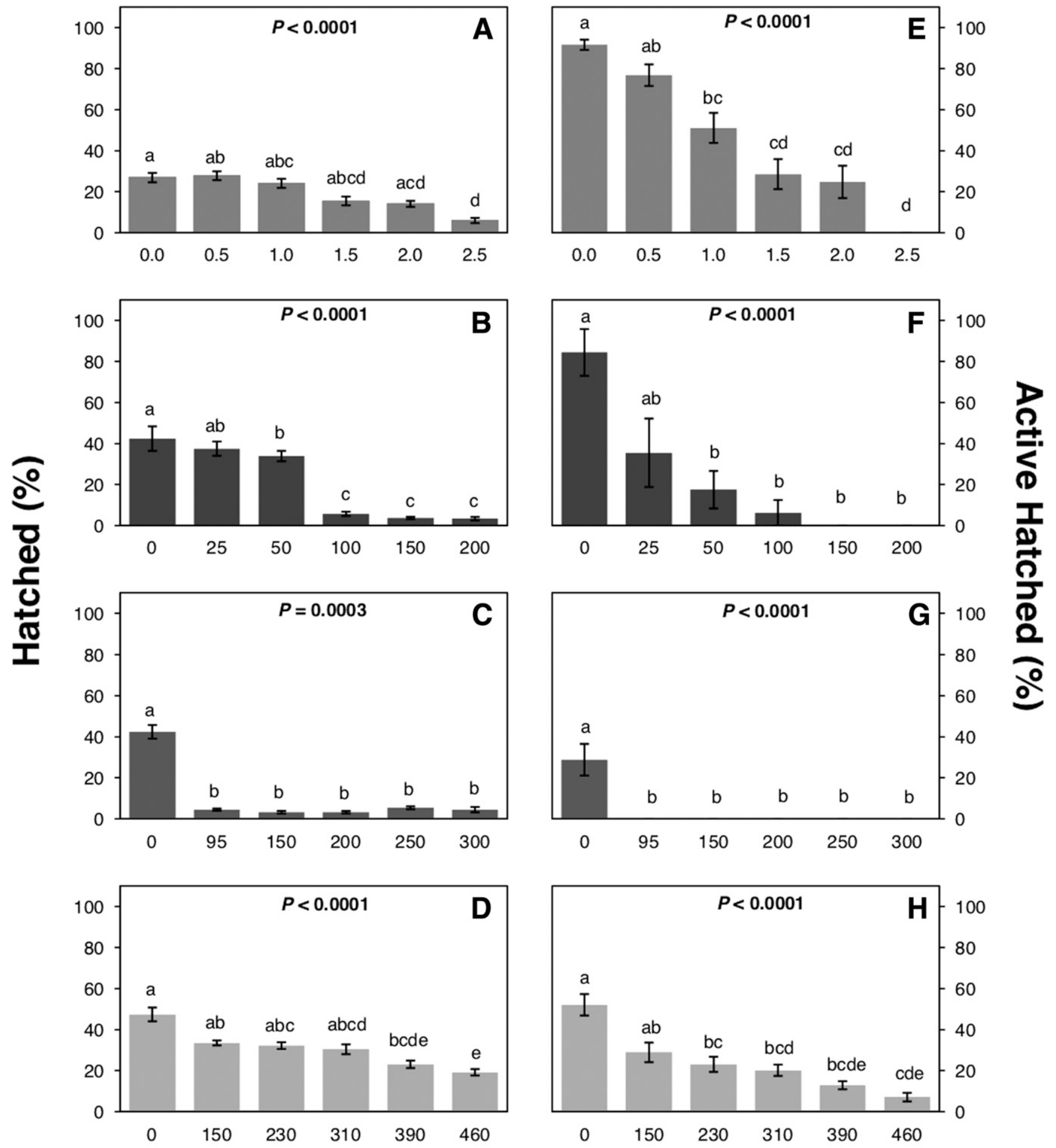

\section{Concentration (ppm)}

Fig. 2. Meloidogyne incognita egg hatch after exposure to nematicides. M. incognita eggs were exposed to $\mathbf{A}$ and $\mathbf{E}$, fluopyram, $\mathbf{B}$ and $\mathbf{F}$, oxamyl, $\mathbf{C}$ and $\mathbf{G}$, fluensulfone, and $\mathbf{D}$ and $\mathbf{H}$, fluazaindolizine for 7 days. The percentage of hatched second-stage juveniles (J2) (graphs on the left) and the percentage of the hatched $\mathrm{J} 2$ that were still active calculated for each compound (graphs on the right) are shown. Columns are the mean of eight replicates; error bars represent the standard error. Data within a graph that share a letter are not significantly different $(P \leq 0.05)$ based on the Kruskal-Wallis test and mean separation using the Wilcox rank sum comparisons test with a Bonferroni multiple comparison correction. 
460 ppm $(P<0.0001 ;$ Fig. 2D). At these two highest concentrations, egg hatch was reduced by 51 and $59 \%$, respectively, compared with the water control. Fluazaindolizine had more of an effect on the mean percentage of active hatched $M$. incognita $\mathrm{J} 2$, with significant differences from the water control at all concentrations tested, except at $150 \mathrm{ppm}(P=0.034$; Fig. $2 \mathrm{H})$.

Motility assays. Motility of $M$. incognita $\mathrm{J} 2$ exposed to $\mathrm{ED}_{50}$ doses of oxamyl, fluopyram, fluazaindolizine, and fluensulfone did not differ from the water control at 2 and $24 \mathrm{~h}$ of exposure (Figs. 3A and C). However, differences were observed with fluensulfone and fluazaindolizine in the percentage of active $M$. incognita $\mathrm{J} 2$ recovered (Fig. 3B and D). At both 2 and $24 \mathrm{~h}$ of exposure to fluensulfone, there was a 61 and $98 \%$ reduction in the percentage of active $M$. incognita $\mathrm{J} 2$, respectively, compared with the water control $(P<0.0001)$. Fluazaindolizine resulted in a 42 and $91 \%$ reduction in the percentage of active $M$. incognita $\mathrm{J} 2$ at 2 and $24 \mathrm{~h}$ of exposure compared with the water control, respectively $(P<$ $0.0001)$. Oxamyl and fluopyram had no effect on the percentage of active $M$. incognita $\mathrm{J} 2$ compared with the water control at either exposure period (Fig. 3B and D).

Infectivity assays. When nematicides were applied at sublethal doses, only fluopyram was unable to prevent reproduction of $M$. incognita (Fig. 4A), with rates of reproduction similar to the untreated control $(P=1.0)$. The other nematicides had a mean RF of 0 and were significantly different from the untreated control $(P<0.0001)$.

Plants treated with fluensulfone had the lowest mean root weight $(0.31 \pm 0.03 \mathrm{~g})$ compared with root weights of the untreated control and fluopyram $(P<0.001$; Fig. 4B). Fluopyram was the only treatment that had similar root weights to that of the untreated control $(P=0.94)$. Root weights were reduced by 56,51 , and $35 \%$

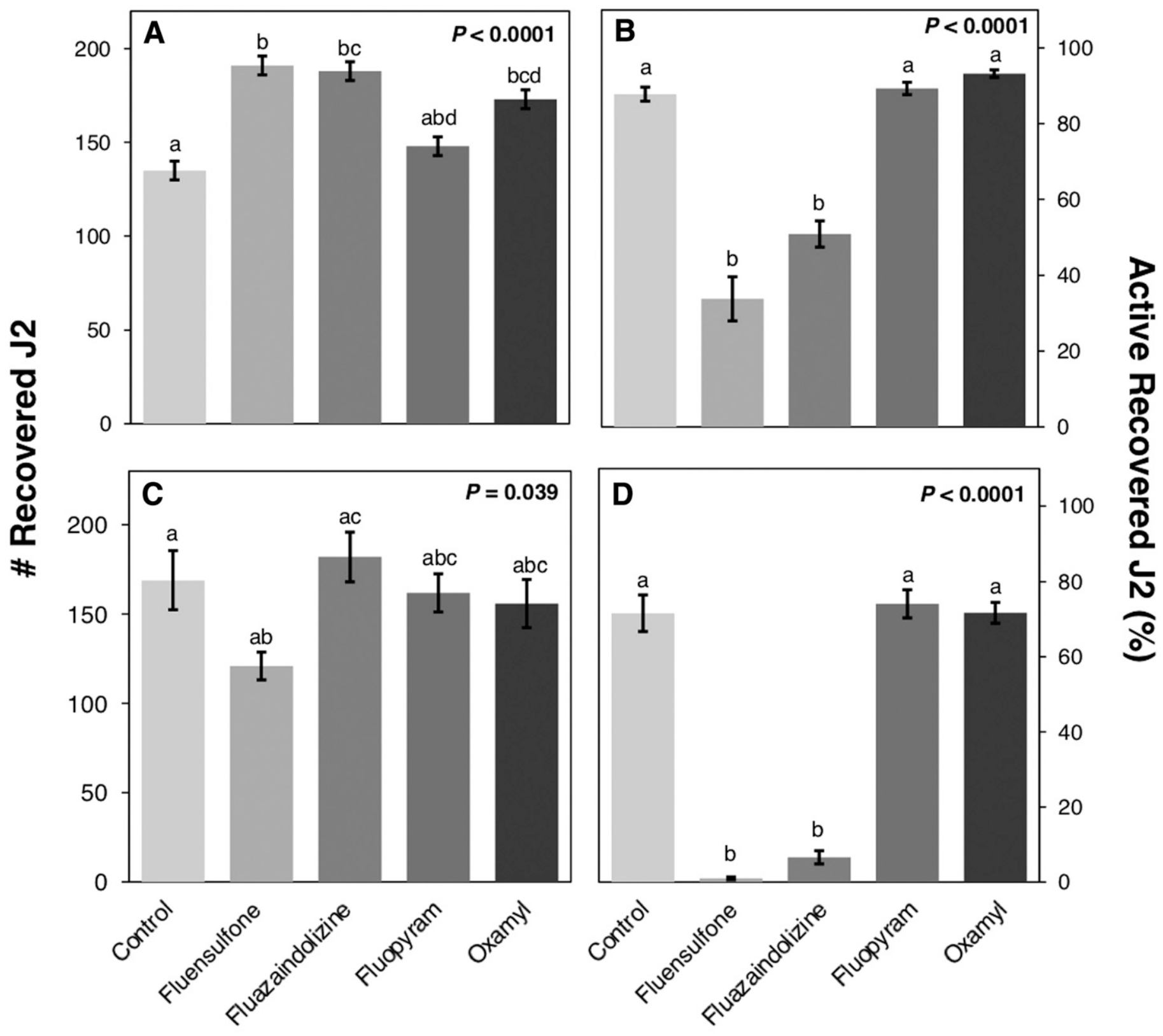

Nematicide

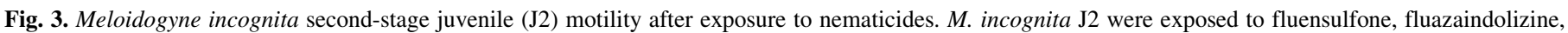

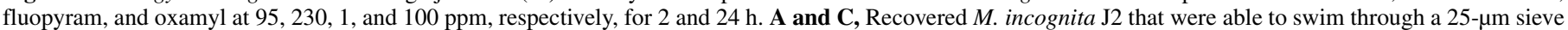

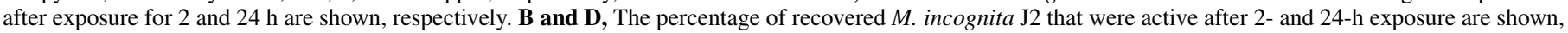

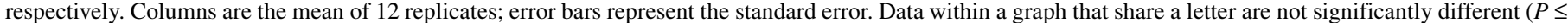
0.05 ) based on the Kruskal-Wallis test and mean separation using the Wilcox rank sum comparisons test with a Bonferroni multiple comparison correction. 
for fluensulfone, fluazaindolizine, and oxamyl, respectively, compared with the untreated control.

\section{DISCUSSION}

M. incognita is a major global pest, with few effective chemical controls available. Of the new nematicides to come onto the market, there have been few published side-by-side comparisons as well as comparisons to industry standards. This study is the first comprehensive evaluation of $M$. incognita activity, motility, and fecundity after exposure to the traditional nematicide, oxamyl, as well as three new nematicides, fluazaindolizine, fluopyram, and fluensulfone. There are many factors that affect persistence and effective concentrations of nematicides in soil, leading to an environment where PPNs are often exposed to sublethal doses of nematicides. Continual exposure to sublethal doses of nematicides could lead to selection of PPNs that are resistant to nematicides, limiting the resources available to manage these pests, which is a profound problem in other phytopathogen systems. Identifying sublethal doses of new nonfumigant nematicides will set the stage for future studies to identify modes of action, cellular effects, effects on gene expression, and possible insights into resistance development.

Oxamyl, a compound used to effectively manage $M$. incognita on a variety of hosts, has traditionally been thought to be a nematistatic compound (McGarvey et al. 1984). M. incognita J2 were able to recover from exposure to 4,000 ppm of oxamyl for $10 \mathrm{~min}$, when placed in water for $24 \mathrm{~h}$ after exposure (McGarvey et al. 1984). In this study, the effect of oxamyl on $M$. incognita activity, motility, and fecundity was variable. Oxamyl was nematicidal as shown in the dose-response and fecundity assays. When exposed to a range of oxamyl concentrations (50 to $140 \mathrm{ppm}$ ) for $24 \mathrm{~h}$ and then placed in water for an additional $24 \mathrm{~h}, M$. incognita $\mathrm{J} 2$ were unable to recover. Moreover, oxamyl behaved as a true nematicide, with $M$. incognita exposed to a sublethal dose of oxamyl unable to reproduce on tomato. However, in the motility assays, $M$. incognita $\mathrm{J} 2$ were able to move and survive after exposure to oxamyl for 2 or $24 \mathrm{~h}$, indicating that oxamyl was nematistatic similar to what was observed by McGarvey et al. (1984). A future experiment looking at rates of movement after nematicide exposure could be beneficial to determine when impact on movement/motility occurs. Oxamyl was also a good ovicide at higher doses, with an $86 \%$ reduction of hatch when eggs were exposed to 100 ppm of oxamyl.

Fluensulfone, a nematicide registered in 2014, behaved like a true nematicide in our short-term exposure assays. $M$. incognita J2 inactivity continued to increase after fluensulfone was removed and replaced with water even at low concentrations. At $25 \mathrm{ppm}$, inactivity increased by $60 \%$ once fluensulfone was removed and replaced with water. Fluensulfone was also an effective ovicide, with a $90 \%$ reduction in hatch at the lowest concentration of fluensulfone tested. Oxamyl and fluensulfone had similar ranges of effective concentrations with similar $\mathrm{ED}_{50}$ values of 100 and 95 ppm, respectively. However, unlike oxamyl, fluensulfone had no effect on nematode motility at 2 and $24 \mathrm{~h}$ of exposure, but activity of motile nematodes was severely affected. This pattern of inactivity followed that observed in the microwell assays; once $M$. incognita $\mathrm{J} 2$ were exposed to this nematicide even at low doses, they were unable to recover. This was further exemplified when $M$. incognita $\mathrm{J} 2$ were exposed to a sublethal dose of fluensulfone in soil and were unable to reproduce on a susceptible host. It is important to note that fluensulfone may be phytotoxic. Fluensulfone had the lowest root biomass of all treatments. This could be an indication of phytotoxicity of this compound or root damage caused by early nematode feeding. Castillo et al. (2017) did not find tomato vigor or yield to be affected when fluensulfone was drip applied at a midlabel rate of 5.85 liter/ha. Further evaluation of the effects of fluensulfone on plant vigor are required.

Fluazaindolizine, a nematicide not yet registered by the U.S. Environmental Protection Agency, has no literature available about its efficacy in a greenhouse or field setting against $M$. incognita or
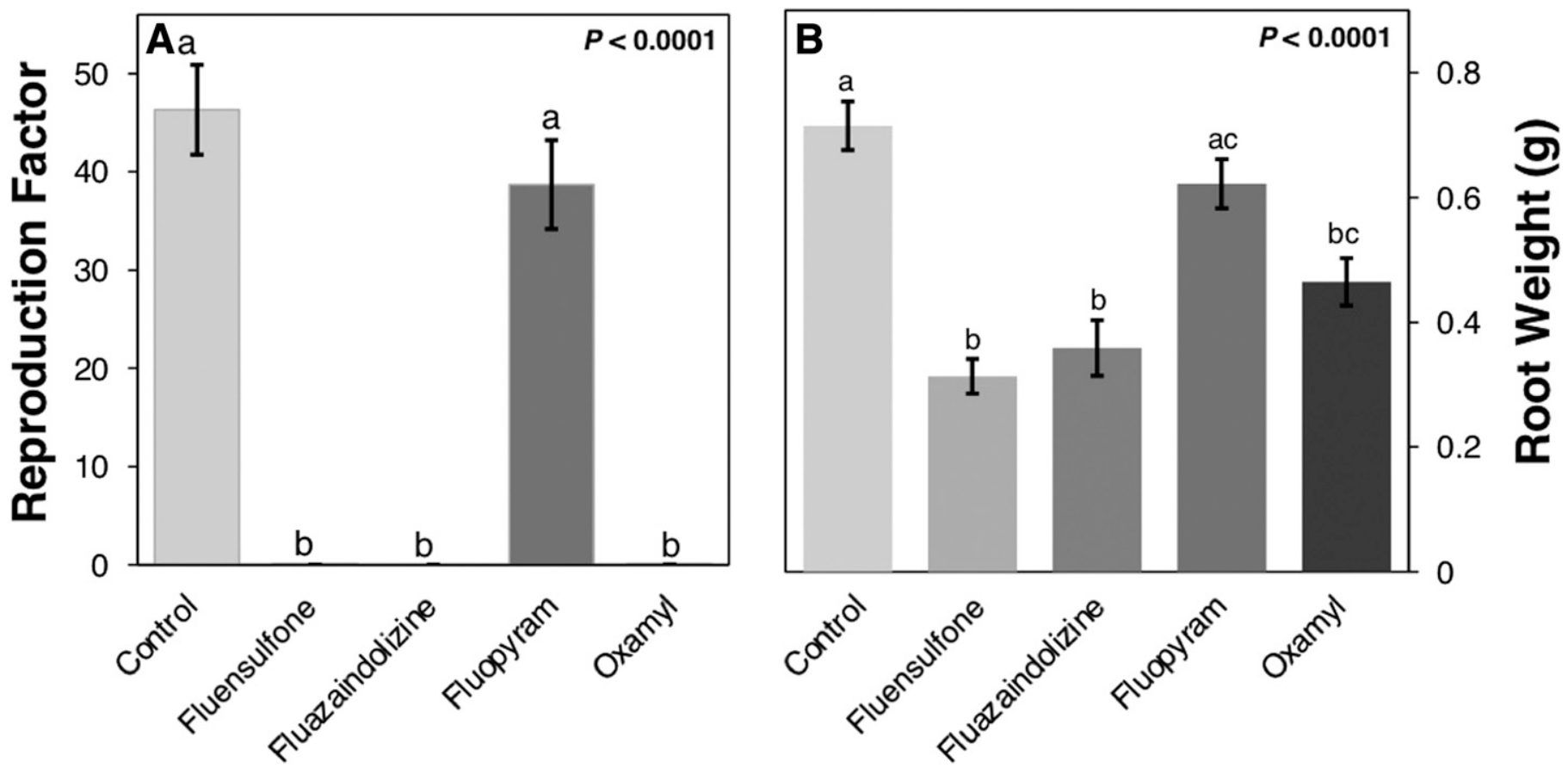

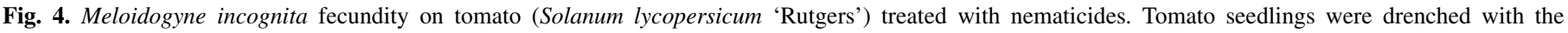

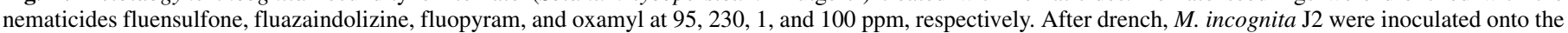

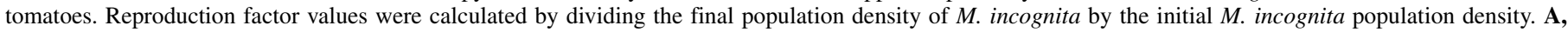

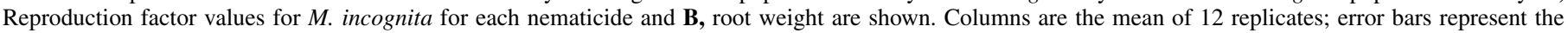

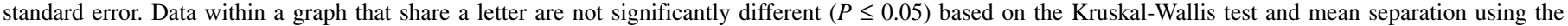
Wilcox rank sum comparisons test with a Bonferroni multiple comparison correction. 
other PPNs. In our short-term assays, it behaved as a true nematicide against $M$. incognita. $M$. incognita $\mathrm{J} 2$ were unable to recover after exposure to fluazaindolizine. Of all the nematicides tested, fluazaindolizine required the highest concentration to be effective. Fluazaindolizine had a $24-\mathrm{h} \mathrm{ED}_{50}$ of $230 \mathrm{ppm}$, which was more than twice that of oxamyl. $M$. incognita $\mathrm{J} 2$ exposed to even high doses of fluazaindolizine did not have high rates of inactivity at $24 \mathrm{~h}$ of exposure. However, removal of the compound did not result in an increase in active $\mathrm{J} 2$; rather, there was an increase in inactivity between 13 to $31 \%$ for all concentrations tested. Unlike fluensulfone, fluazaindolizine had little effect on egg hatch. Only at 390 and 460 ppm of fluazaindolizine was egg hatch significantly reduced. Similar to the other nematicides tested, $M$. incognita J2 motility was not affected by fluazaindolizine; but like fluensulfone, activity of the motile $M$. incognita J2 was significantly affected. Motile M. incognita $\mathrm{J} 2$ exposed to fluazaindolizine for 2 and $24 \mathrm{~h}$ had inactivity rates of 50 and $94 \%$, respectively. Combined with the effects of fluazaindolizine shown in the dose-response curves, fluazaindolizine is a slower-acting nematicide compared with fluensulfone. This is consistent with results by Lahm et al. (2017), in which M. incognita $\mathrm{J} 2$ exposed to low doses (5 to $50 \mathrm{ppm}$ a.i.) of fluazaindolizine showed no symptoms in the first hours after exposure but ultimately died 24 to $96 \mathrm{~h}$ later. Fluazaindolizine was very effective at preventing reproduction of $M$. incognita on tomato even when soil was treated with a sublethal dose.

Fluopyram was the only nematicide found to be nematistatic in these short-term exposure assays. At all concentrations evaluated ( 0.5 to $4 \mathrm{ppm}$ ), at least $80 \%$ of $M$. incognita $\mathrm{J} 2$ were able to recover from exposure. Faske and Hurd (2015) demonstrated that $M$. incognita $\mathrm{J} 2$ exposed to fluopyram at $5.58 \mathrm{ppm}$ for $2 \mathrm{~h}$ reduced mobility by $80 \%$. However, their results also showed a $58 \%$ recovery of mobility after removal of the nematicide, consistent with $M$. incognita $\mathrm{J} 2$ recovery shown in this study. Faske and Hurd (2015) also determined a 24-h ED 50 of 1.18 ppm and a $24-\mathrm{h} \mathrm{ED}_{90}$ of $5.31 \mathrm{ppm}$ compared with the predicted $\mathrm{ED}_{50}$ and $\mathrm{ED}_{90}$ of 1.30 and $4.85 \mathrm{ppm}$ found in this study, respectively. Egg hatch was unaffected by all but one concentration tested, making fluopyram a poor ovicide against $M$. incognita. Fluopyram had no effect on the motility of nematodes and little impact on the activity of nematodes that were motile. This again reflects the nematistatic nature of this compound. Fluopyram also did not suppress reproduction of $M$. incognita on tomato when soil was drenched with a sublethal dose. This is in contrast to a $31 \%$ reduction in root galling of tomato compared with the water control when $M$. incognita J2 were exposed to $1.3 \mathrm{ppm}$ of fluopyram for $2 \mathrm{~h}$ and inoculated onto tomato (Faske and Hurd 2015).

Several studies have evaluated these new nematicides in greenhouse, microplot, and field trials. Fluopyram, oxamyl, and fluensulfone have been reported as effective nematicides for controlling $M$. incognita in lima beans, both in greenhouse trials and microplots (Jones et al. 2017). Lima beans treated with oxamyl at a rate of 2.25 liter a.i./ha reduced $M$. incognita galling by 10 to $70 \%$. In this same trial, fluopyram seemed to perform just as well as oxamyl at a rate of 0.22 liter a.i./ha, with a reduction in $M$. incognita galling on lima beans of 55 and $64 \%$. Fluensulfone performed the best, with a 91 and $81 \%$ reduction in $M$. incognita galling on lima beans when applied at 2.34 liter a.i./ha. These results (Jones et al. 2017) support the findings of this study, in that oxamyl and fluensulfone are both strong nematicides and effective treatments for M. incognita. The results of Jones et al. (2017) also indicate that in order for fluopyram to be an effective nematicide, dosage is important, with control observed in the lima bean study, but none found in this study when a sublethal dose of fluopyram was used. Jones et al. (2017) also indicated that fluensulfone had potentially phytotoxic effects at the 2.34 liter a.i./ha rate of application in a microplot trial where there was a $14 \%$ reduction in lima bean emergence, consistent with the low tomato root weights shown in this study.
When fluensulfone was applied at a rate of $3.0 \mathrm{~kg}$ a.i./ha through drip-pulse irrigation to cucumber, there was a $73 \%$ reduction in $M$. incognita $\mathrm{J} 2$ population densities along with a reduction in root galling (Morris et al. 2016). In growth chamber trials conducted in the same study (Morris et al. 2016), fluensulfone was applied foliarly to tomato at rates of 3,000, 6,000 and 12,000 ppm; an additional treatment in the study was foliar applied oxamyl at a rate of $4,800 \mathrm{ppm}$. All of the treatments reduced root galling by $M$. incognita and the number of females in the root system compared with an untreated control, but the treatments were unable to eliminate $M$. incognita reproduction. Oxamyl was the most effective treatment in reducing $M$. incognita females and galling except in comparison with the highest dosage of fluensulfone. In this study, when drenching the soil at rates a fraction of those used by Morris et al. (2016), oxamyl at $100 \mathrm{ppm}$ and fluensulfone at 95 ppm both eliminated reproduction of $M$. incognita. This finding, along with the success of drip application of fluensuflone in another study (Morris et al. 2016), suggests that fluensulfone works best when applied to the root system.

Fluopyram applied at the labeled rate $(249 \mathrm{~g}$ a.i./ha) to the surface of tomato plants 2 days postinoculation with $M$. incognita eggs had no effect on the number of $M$. incognita eggs per gram of root compared with an untreated control (Heiken 2017). These results support those found in this study; fluopyram was a poor nematicide when applied as a soil drench at low doses. However, Heiken (2017) found that when fluopyram was applied at the labeled rate 2 weeks after tomato plants were inoculated with $M$. incognita eggs, fluopyram was able to reduce the number of eggs per gram of root from 16,000 to $<1,500$ compared with the untreated control. In the present study, fluopyram was a poor ovicide, with the only effects on $M$. incognita egg hatch observed at the highest concentration tested. The timing and stage of $M$. incognita present when treatment is applied may be critical to the efficacy of this nematicide.

Overall, the newly developed nematicides, fluensulfone and fluazaindolizine were comparable in their efficaciousness against $M$. incognita to the traditionally used nematicide oxamyl. Even at sublethal doses, these three nematicides suppressed $M$. incognita in fecundity assays. However, the effects of these compounds on plant vigor remain unclear and warrant further exploration. All of the nematicides tested had limited effects on short-term $M$. incognita $\mathrm{J} 2$ motility, but this did not affect the ability of these compounds to protect against nematode infection. In this study, fluopyram was the least effective nematicide against $M$. incognita. Fluensulfone proved to be the only ovicidal compound at sublethal doses, with oxamyl having ovicidal activity at higher concentrations. The dose-response curves developed in this study will be beneficial in future research, as the $\mathrm{ED}_{50}$ doses determined could be used to explore the broader effects of nematicides on $M$. incognita biology and potentially understanding the modes of action of fluazaindolizine and fluensulfone.

\section{LITERATURE CITED}

Bernard, G. C., Egnin, M., and Bonsi, C. 2017. The impact of plant-parasitic nematodes on agriculture and methods of control. In: Nematology: Concepts, Diagnosis and Control. InTech Open, London.

CABI, 2018. Meloidogyne incognita. In: Invasive Species Compendium. CAB International, Wallingford, UK. www.cabi.org/isc

Castillo, G. X., Ozores-Hampton, M., and Navia Gine, P. A. 2017. Effects of fluensulfone combined with soil fumigation on root-knot nematodes and fruit yield of drip-irrigated fresh-market tomatoes. Crop Prot. 98:166-171.

Costa, L. G., Giordano, G., Guizzetti, M., and Vitalone, A. 2008. Neurotoxicity of pesticides: A brief review. Front. Biosci. 13:1240-1249.

Duniway, J. M. 2002. Status of chemical alternatives to methyl bromide for pre-plant fumigation of soil. Phytopathology 92:1337-1343.

Faske, T. R., and Hurd, K. 2015. Sensitivity of Meloidogyne incognita and Rotylenchulus reniformis to fluopyram. J. Nematol. 47:316-321.

Haydock, P. P. J., Ambrose, E. L., Wilcox, A., and Deliopoulos, T. 2012. Degradation of the nematicide oxamyl under field and laboratory conditions. Nematology 14:339-352.

Heiken, J. A. 2017. The Effects of Fluopyram on Nematodes. North Carolina State University, Raleigh. 
Jones, J. G., Kleczewski, N. M., Desaeger, J., Meyer, S. L. F., and Johnson, G. C. 2017. Evaluation of nematicides for southern root-knot nematode management in lima bean. Crop Prot. 96:151-157.

Jones, J. T., Haegeman, A., Danchin, E. G. J., Gaur, H. S., Helder, J., Jones, M. G. K., et al. 2013. Top 10 plant-parasitic nematodes in molecular plant pathology. Mol. Plant Pathol. 14:946-961.

Kearn, J., Lilley, C., Urwin, P., O'Connor, V., and Holden-Dye, L. 2017. Progressive metabolic impairment underlies the novel nematicidal action of fluensulfone on the potato cyst nematode Globodera pallida. Pestic. Biochem. Physiol. 142:83-90.

Kearn, J., Ludlow, E., Dillon, J., O'Connor, V., and Holden-Dye, L. 2014. Fluensulfone is a nematicide with a mode of action distinct from anticholinesterases and macrocyclic lactones. Pestic. Biochem. Physiol. 109:44-57.

Lahm, G. P., Desaeger, J., Smith, B. K., Pahutski, T. F., Rivera, M. A., Meloro, T., et al. 2017. The discovery of fluazaindolizine: A new product for the control of plant parasitic nematodes. Bioorg. Med. Chem. Lett. 27: $1572-1575$.

Ludlow, K. 2015a. Public release summary on the evaluation of the new active fluopyram in product Luna Privilege fungicide. Australian Pesticides and Veterinary Medicines Authority. https://apvma.gov.au/sites/default/files/ publication/14166-prs-fluopyram.pdf

Ludlow, K. 2015b. Public release summary on the evaluation of the new active fluensulfone in product NIMITZ 480 EC nematicide. Australian Pesticides and Veterinary Medicines Authority. http://sk.sagepub.com/reference/ nanoscience/n22.xml

Martin, F. N. 2003. Development of alternative strategies for management of soilborne pathogens currently controlled with methyl bromide. Annu. Rev. Phytopathol. 41:325-350.

McGarvey, B. D., Potter, J. W., and Chiba, M. 1984. Nematostatic activity of oxamyl and N,N-dimethyl-1-cyanoformamide (DMCF) on Meloidogyne incognita juveniles. J. Nematol. 16:328-331.

Meyer, S. L., Lakshman, D. K., Zasada, I. A., Vinyard, B. T., and Chitwood, D. J. 2008. Dose-response effects of clove oil from Syzygium aromaticum on the root-knot nematode Meloidogyne incognita. Pest Manag. Sci. 64:223-229.

Mitkowski, N. A., and Abawi, G. S. 2003. Root-knot nematodes. Plant Health Instructor. doi.org/10.1094/PHI-I-2003-0917-01

Morris, K. A., Langston, D. B., Davis, R. F., Noe, J. P., Dickson, D. W., and Timper, P. 2016. Efficacy of various application methods of fluensulfone for managing root-knot nematodes in vegetables. J. Nematol. 48:65-71.
Morris, K. A., Langston, D. B., Dickson, D. W., Davis, R. F., Timper, P., and Noe, J. P. 2015. Efficacy of fluensulfone in a tomato-cucumber double cropping system. J. Nematol. 47:310-315.

Nitao, J. K., Meyer, S. L., and Chitwood, D. J. 1999. In-vitro assays of Meloidogyne incognita and Heterodera glycines for detection of nematodeantagonistic fungal compounds. J. Nematol. 31:172-183.

Noling, J. W. 1997. Movement and toxicity of nematicides in the plant root zone. http://edis.ifas.ufl.edu/ng002

Oka, Y. 2014. Nematicidal activity of fluensulfone against some migratory nematodes under laboratory conditions. Pest Manag. Sci. 70:1850-1858.

Osman, K. A., Al-Rehiayani, S. M., Al-Deghairi, M. A., and Salama, A. K. 2009. Bioremediation of oxamyl in sandy soil using animal manures. Inter. Biodeter. Biodegrad. 63:341-346.

Perry, R. N., and Moens, M., eds. 2013. Plant Nematology, 2nd ed. CAB International, Oxfordshire, UK.

R Core Team. 2017. R: A Language and Environment for Statistical Computing. R Foundation for Statistical Computing, Vienna, Austria.

Ritz, C., Baty, F., Streibig, J. C., and Gerhard, D. 2015. Dose-response analysis using R. PLoS One 10:e0146021.

Trudgill, D. L., and Blok, V. C. 2001. Apomictic, polyphagous root-knot nematodes: Exceptionally successful and damaging biotrophic root pathogens. Annu. Rev. Phytopathol. 39:53-77.

U.S. Environmental Protection Agency. 2008. Fenamiphos: Amendment to use deletion and product cancellation order. Fed. Regist. 73:33082-33084. https://www.federalregister.gov/documents/2008/06/11/E8-13003/fenamiphos-amendment-to-use-deletion-and-product-cancellation-order

Windham, G. L., and Williams, W. P. 1987. Host suitability of commercial corn hybrids to Meloidogyne arenaria and M. incognita. J. Nematol. 19: 13-16.

Yates, S. R., Gan, J., Papiernik, S. K., Dungan, R., and Wang, D. 2002. Reducing fumigant emissions after soil application. Phytopathology 92: 1344-1348.

Zasada, I. A., Halbrendt, J. M., Kokalis-Burelle, N., LaMondia, J., McKenry, M. V., and Noling, J. W. 2010. Managing nematodes without methyl bromide. Annu. Rev. Phytopathol. 48:311-328.

Zasada, I. A., Klassen, W., Meyer, S. L., Codallo, M., and Abdul-Baki, A. A. 2006. Velvetbean (Mucuna pruriens) extracts: Impact on Meloidogyne incognita survival and on Lycopersicon esculentum and Lactuca sativa germination and growth. Pest Manag. Sci. 62:1122-1127. 